\title{
Correlation between Chondromalacia Patella and Patellofemoral Factors in Middle-Age Population: A Clinical, Functional, and Radiological Analysis
}

\author{
Rashmeet Kaur ${ }^{1}$ Anshul Dahuja ${ }^{2} \quad$ Chandanpreet Kaur $^{3} \quad$ Jagdeep Singh ${ }^{2}$ Paramdeep Singh ${ }^{1}$ \\ Radhe Shyam² \\ 1Department of Radiodiagnosis, Guru Gobind Singh Medical \\ College, Faridkot, Punjab, India \\ 2Department of Orthopaedics, Guru Gobind Singh Medical College, \\ Faridkot, Punjab, India \\ ${ }^{3}$ Department of Physical Medical Rehabilitation, Guru Gobind Singh \\ Medical College, Faridkot, Punjab, India

\begin{abstract}
Address for correspondence Anshul Dahuja, Department of Orthopaedics Guru Gobind Singh Medical College, Faridkot 151203, Punjab, India (e-mail: anshuldahuja@gmail.com).
\end{abstract} \\ Indian J Radiol Imaging 2021;31:252-258.
}

\begin{abstract}
Background Despite higher incidence of patellofemoral pain (PFP) and consequently morbidity, the understanding about PF factors leading to PF arthritis is way lacking. Material and Methods A prospective study of first 80 patients who were diagnosed with chondromalacia patella (CMP) on magnetic resonance imaging (MRI) divided into early and late CMP groups were evaluated clinically, radiologically, and in terms of functional outcome.

Results : Quadriceps angle, Clark's test, and Insall-Salvati ratio results were nonsignificant despite greater values were observed in late CMP group, whereas trochlear morphology results (sulcus angle: 153:138 degrees and sulcus depth 3.9:5.4 mm) and

Keywords

- chondromalacia patella

- patellofemoral

- trochlea clinical scores were significant in late CMP group (Kujala's score: 61:78, whereas PF pain score: 43:25). Type-C patellar morphology was found in greater number in late CMP cohort.

Conclusion Trochlear and patellar morphologies along with clinical scores play a key role in understanding of the CMP.
\end{abstract}

\section{Introduction}

Knee pain is the second most prevailing disorder of knee and patellofemoral pain (PFP) being considered one of the most common forms of knee pain, with incidence ranging between 15 and $45 \%{ }^{1,2}$ PFP is described as nontraumatic diffuse anterior knee pain (AKP) during load bearing activities of the joint such as squatting, running, climbing, and descending stairs. ${ }^{1,2}$ The degeneration of cartilage can quickly lead to tracking issues of the patella and, if left untreated, can often lead to osteoarthritis. ${ }^{3}$ There is no descriptive treatment

published online July 27, 2021
DOI https://doi.org/

10.1055/s-0041-1734361 ISSN 0971-3026 of chondromalacia patella (CMP) due to poor regenerative properties and complex physiology of cartilage. ${ }^{4}$ Patellofemoral joint is a part of the knee joint and ironically even knowing the high incidence of PF arthritis and morbidity in middle aged patients, the literature on the understanding of PF factors is scarce and treatment is neither promising nor clear making it one of the tangled topic in today's orthopaedics. ${ }^{5-9}$

There is very limited research on correlation of stage of CMP on severity of anterior knee pain and resultant functional morbidity. ${ }^{10-14}$ To our knowledge, this is the first study which has taken into account comprehensively the clinical

\section{(C) 2021. Indian Radiological Association.}

This is an open access article published by Thieme under the terms of the Creative Commons Attribution-NonDerivative-NonCommercial-License, permitting copying and reproduction so long as the original work is given appropriate credit. Contents may not be used for commercial purposes, or adapted, remixed, transformed or built upon. (https://creativecommons.org/licenses/by-nc-nd/4.0/).

Thieme Medical and Scientific Publishers Private Ltd. A-12, Second Floor, Sector -2, NOIDA -201301, India 
quadriceps angle ( $Q$ angle), PF indexes, patellar morphology, and clinical scores to be assessed in various grades of CMP. The present study aimed to investigate whether chondromalacia has any association with PF alignment, PF indexes, patellar morphology, knee pain severity, and function along with the hypothesis that clinical and radiological parameters together can better detect and elaborate the CMP patients.

\section{Materials and Methods}

This was a single-center, prospective, cross-sectional study conducted at a high-volume tertiary center in North India after obtaining ethical approval the institutional ethical committee. We examined middle aged patients in the orthopaedic outpatient department (OPD) with anterior knee pain who had undergone clinical and magnetic resonance imaging (MRI) examination of the knee and first 50 patients who were diagnosed with late/advanced CMP (grades III and IV, i.e., cartilage loss) constituted group I and 30 patients as (group II) with early CMP (grades I and II, i.e., no cartilage loss). ${ }^{12}$ Written informed consent was obtained from all patients. Patients with anterior knee pain for longer than a month and worsening of the anterior knee pain, with at least one of the following activities, were included in the study: climbing or descending stairs, squatting, jumping, and prolonged sitting. The study exclusion criteria were a history of a major trauma, history of past lower extremity surgery and/or fracture, presence of neuromuscular disease, inflammatory rheumatic disease, epilepsy, dementia, pregnancy, history of intra-articular injection, genu varus-valgus, difference in leg length and detection of severe ligament, Osgood-Schlatter disease, or advanced stage osteoarthritis in MRI.

In addition to anterior knee pain, complaints, including swelling, feeling of knee giving way, stiffness, locking at the knee, clicking noise during movements of the knee, crepitation, pain triggered by prolonged sitting, first-step limping, sliding knee cap, and difficulty in squatting, were recorded. Patients' knee physical examination findings, Kujala's PF scoring system (KPSS), and functional index questionnaire were obtained from the outpatient clinic records. The $\mathrm{Q}$ angle was measured by two vectors extending from the anterior midpatella to the anterior superior iliac spine (ASIS) and tibial tuberosity with knee in 15-degree flexion. The center of the goniometer was placed at the midpoint of the patella. One arm of the goniometer was aligned with the string leading to the anterior superior iliac spine, and the other arm of the goniometer was aligned with the tibial tubercle. ${ }^{10,11}$

Dedicated knee MRI images were evaluated by a radiologist for staging of chondromalacia and PF indexes. All MRI scans were performed on the $1.5 \mathrm{~T}$ Magnetom Avanto Siemens MRI and with patient in supine position with their knees 15-degree flexion. Five different measurements were performed in all patients' knee MRI images as follows:

1. Trochlear sulcus angle: it is the angle between the two lines that connects the deepest point of the trochlea to the anterior medial and lateral femoral condyles measured on axial scans ${ }^{13}$ (-Fig. 1), a trochlear angle greater than 144 degrees was evaluated as trochlear hypoplasia. ${ }^{4}$
2. Trochlear sulcus depth: it is the distance between the deepest point of the trochlear sulcus and the line connecting medial and lateral femoral condyles. A sulcus depth lower than $5 \mathrm{~mm}$ was accepted as hypoplasia and a depth lower than $3 \mathrm{~mm}$ was accepted as a sign of dysplasia. ${ }^{13}$

3. Insall-Salvati index (patellar tendon length/the longest diagonal diameter of patella): it is the ratio of patellar tendon length to the longest diagonal diameter of patella (-Fig. 2). It was measured using the sagittal section where maximum length of patella was visualized (often the section where anterior cruciate ligament can be visualized). ${ }^{15}$ Patellar tendon length was divided by the longest diagonal diameter to calculate the Insall-Salvati index. Values below 1.1 were accepted as patella baja, and values above 1.3 were accepted as patella alta.

4. Lateral patellofemoral angle: it is the angle between the line connecting anterior medial and lateral femoral condyles and the line drawn tangential to the lateral patellar facet. This angle is used for the evaluation of patellar tilt and is measured using the axial section corresponding to the middle point of the patella at the sagittal plane (-Fig. 3). ${ }^{15}$ This angle usually opens laterally, so any medial angulation or an angle lower than 8 degrees to the lateral side was evaluated as a sign of abnormal tilt. ${ }^{16}$

5. Patellar translation: it is measured to assess patellar subluxation. It is the distance between the vertical line passing from the anterior of medial femoral condyle and the vertical line passing from the medial most border of the patella measured on the axial planes. A $>2-\mathrm{mm}$ sliding toward the lateral side was evaluated as subluxation. ${ }^{17}$

Additionally, in all patients, the CMP grading/stage was determined from the MRI images. Patients were categorized into early and late CMP groups based on four stages on the MRI staging system that was modified Outerbridge's arthroscopy staging system. ${ }^{12,18,19}$ In case, cartilage injuries in the medial and patellar facet were at different stages, the highest stage was considered for categorizing the patient.

Finally, we evaluated respective correlation of the advanced CMP group (stages 3 and 4) and early CMP group with PF indexes, patellar morphology (Wiberg's classification), $\mathrm{Q}$ angle, and patellar position in patients with anterior knee pain. ${ }^{14}$

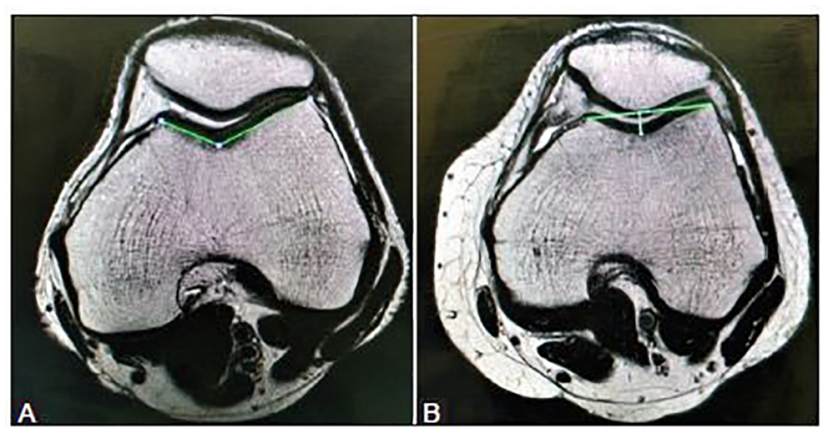

Fig. 1 (A and B) Axial T2-W MRI at the level of trochlear sulcus measuring the corresponding angle and sulcus depth.T2-W, T2-weighted; MRI, magnetic resonance imaging. 


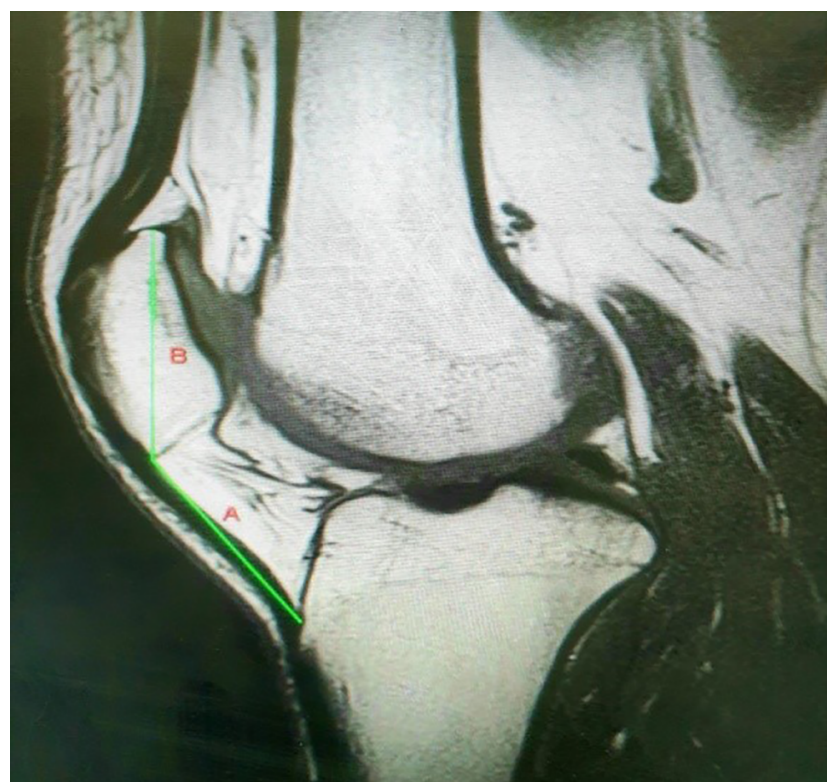

Fig. 2 T2-WI sagittal imaging showing patellar diagonal length (B) and patellar tendon (A) with Insall-Salvati ratio calculated as A/B. T2-WI, T2-weighted image.

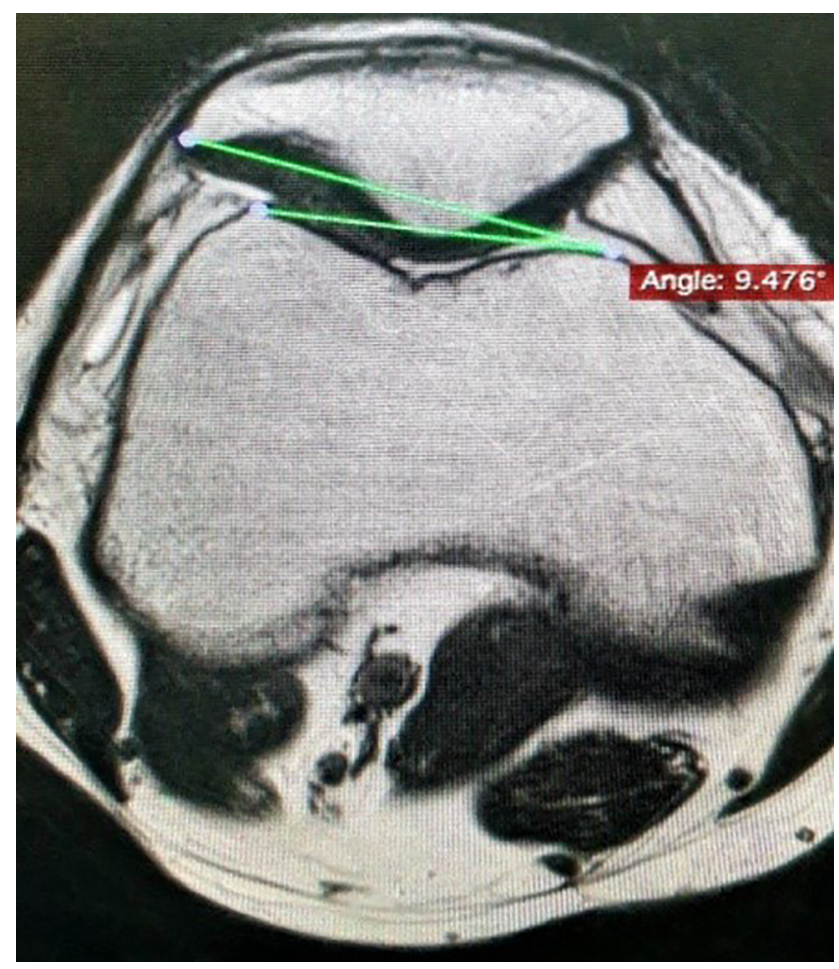

Fig. 3 Axial T2-WI MR showing the lateral patellofemoral angle. MR, magnetic resonance; T2-WI, T2-weighted image.

To test the interobserver reliability, another radiologist performed quantitative and semiquantitative measurements of randomly selected 50 knees among 80 knees.

\section{Statistical Analysis}

Statistical analysis will be performed using the Statistical Package for Social Sciences (SPSS) software version 16 (SPSS Inc., Chicago, Illinois, United States). To compare the

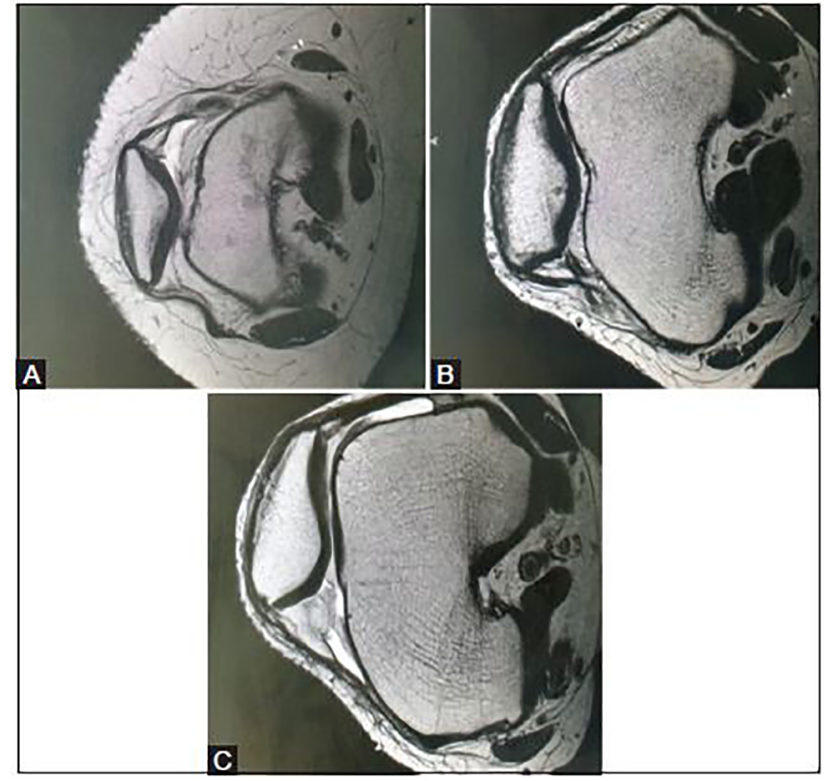

Fig. 4 (A) MRI axial images assessing types of patellar morphology based on Wiberg's classification. Type A showing symmetrical sized concave facets. (B) MRI axial images assessing types of patellar morphology based on Wiberg's classification. Type B showing intermediate type (less concave). (C) MRI axial images assessing types of patellar morphology based on Wiberg's classification, type $C$ with more vertically oriented and smaller medial facet. MRI, magnetic resonance imaging.

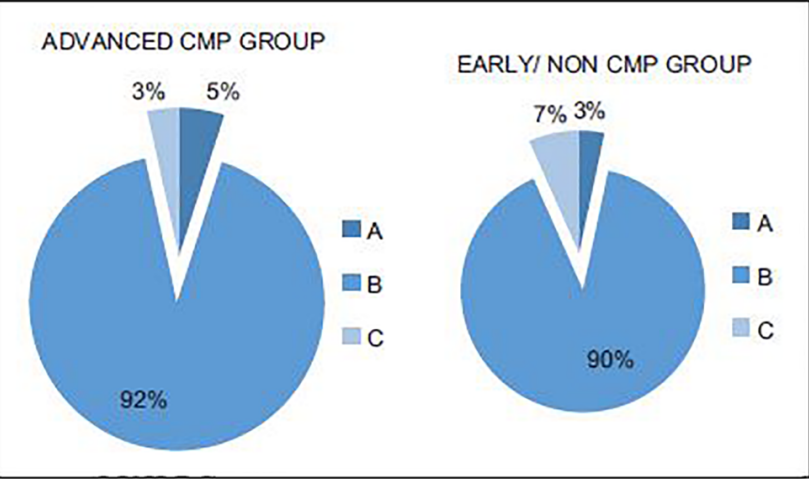

Fig. 5 Pie chart comparison between two groups based on Wiberg's classification with no significant preponderance to a particular type of patellar morphology.

categorical data in terms of other categorical properties, Chi-square and Fisher's exact tests were used. Any two groups were compared with regard to measurement values using the Mann-Whitney $U$-test. A $p$-value of $<0.05$ was accepted as statistically significant.

\section{Results}

Only a small fraction of patients showed type-A and $-C$ morphologies of patella as shown in $\boldsymbol{- F i g s .} \mathbf{4}$ and $\mathbf{5}$. Trochlear morphology results came out to be significant with higher sulcus angle (153 degrees) and shallow trochlear sulcus $(3.9 \mathrm{~mm})$ as shown in - Figs. 1 and $\mathbf{6}$. We observed higher 




Fig. 6 : Pictoral representation for comparison of (A) trochlear sulcus angle in late and early CMP group with significant raised values in late CMP group. (B) Showing rest of patellofemoral indices in late and early CMP group. CMP, chondromalacia patella.

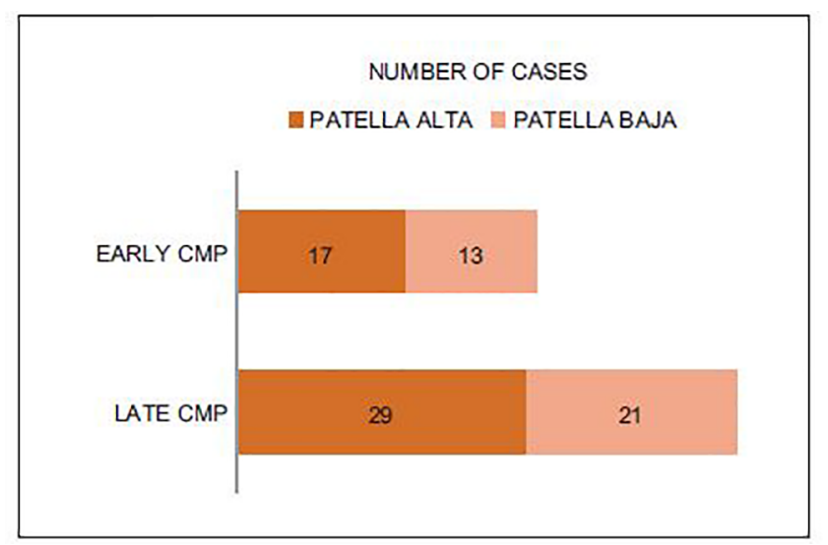

Fig. 7 Bar charts to compare the effective pain scores in late and early CMP groups, the Kujala's pain score indicates more painful process in late CMP group with lower scores, meanwhile the patellofemoral pain system indicates more pain with higher score for late CMP group. CMP, chondromalacia patella.

number of patients in patella alta:patella baja in late CMP group but the results are not significant. Clinical scores were significantly better in early CMP group as compared with late CMP group (-Fig. 7).

\section{Discussion}

In previous studies, the overall sensitivity and specificity of MRI in the diagnosis of patellar cartilage defects ranged between 83 and $97 \% .^{15}$ Only $10 \%$ of CMP patients require surgery, so it is very difficult to justify every doubtful CMP patient to prepare for surgery. ${ }^{16}$

A high Q-angle leads to valgus stress with excessive pressure on PF articulation, consequently resulting in CMP. Most of the western studies suggest that there is a significant association between AKP and Q-angle ${ }^{17-19}$ and there are multiple studies to counter the same. ${ }^{18,20}$ So, the validity of Q-angle is dubious. In our study, the average Q-angle in advanced and early CMP group was 17.1 and 13.2 degrees favoring association between Q-angle and anterior knee pain but the result was statistically nonsignificant (as shown in - Table $\mathbf{1}$ ). We believe that $\mathrm{Q}$-angle is an important parameter which can have a significant association with anterior knee pain but the cause of indecisive results could be because of its measurement bias, dynamic character, and multifactorial association.

To evaluate the relationship between the stage of chondromalacia and severity of pain experienced by patients, PFP severity scale scores were used. ${ }^{5-7}$ Patients in the advanced-stage CMP group had higher scores. To our knowledge, there are only couple of studies in literature which analyzed the relationship between pain intensity and disease progression. ${ }^{6,21,22}$ In literature, only one study prospectively followed-up clinical symptoms in patients who were categorized based on trochlear and patellar cartilage injury in arthroscopy and found greater functional limitation and more subjective complaints among patients with more severe cartilage injury. ${ }^{6}$ Our study showed a significant difference in the clinical scores of late and early CMP groups with Kujala's functional score lower (61 as compared with 78 in early CMP) and PF pain score higher in the advanced CMP group (43 in comparison to 25 in early CMP shown in - Table 2). Our results equate with Aysin et al who observed that those with more advanced CMP reported higher pain severity and lower knee function compared with those with early CMP, but they found no difference in the MRI measurements of two groups. ${ }^{21}$

Table 1 Demographics of patients with anterior knee pain

\begin{tabular}{|c|c|c|}
\hline Patient profile & Late CMP (50) & Early CMP (30) \\
\hline Age (range; y) & $44(38-53)$ & $41(36-50)$ \\
\hline M:F ratio & $29: 21$ & 19:11 \\
\hline Average weight (kg) & $71.8 \mathrm{~kg}$ & 69.9 \\
\hline Unilateral:bilateral & $36: 14$ & $21: 9$ \\
\hline Average $Q$ angle & 17.1 & 13.2 \\
\hline $\begin{array}{l}\text { ROM of knee joint } \\
\text { (degree) }\end{array}$ & $0-105$ & $0-120$ \\
\hline $\begin{array}{l}\text { Any swelling/ } \\
\text { synovitis }\end{array}$ & 6 & 1 \\
\hline $\begin{array}{l}\text { Pain on specific } \\
\text { activity }\end{array}$ & 29 & 19 \\
\hline Squatting & 22 & 2 \\
\hline $\begin{array}{l}\text { Climbing on } \\
\text { stairs }\end{array}$ & 13 & 0 \\
\hline First-step pain & & \\
\hline
\end{tabular}

Abbreviations: CMP, chondromalacia patella; F, female; M, male; $Q$ angle, quadriceps angle; ROM, range of motion; 
Table 2 Comparison of clinical examination and patellofemoral scores in advanced CMP and non-CMP group

\begin{tabular}{|l|l|l|l|}
\hline Assessment & Late CMP & Early CMP & $\boldsymbol{P}$ \\
\hline Clark test (+ve) & $23 / 50$ & $11 / 30$ & 0.1 \\
\hline Average Q angle (degree) & 17.1 & 13.2 & 0.1 \\
\hline Kujala's patellofemoral scoring system (0-100) & 61 & 78 & 0.009 \\
\hline Patellofemoral pain scale (0-80) & 43 & 25 & 0.007 \\
\hline
\end{tabular}

Abbreviations: CMP, chondromalacia patella; Q angle, quadriceps angle.

Table 3 MRI assessment of patellofemoral indexes and their comparison in advanced CMP and early CMP groups

\begin{tabular}{|l|l|l|l|}
\hline Patellofemoral indexes & Late CMP group & Early CMP Group & $p$ \\
\hline Trochlear sulcus angle (degree) & 153 & 138 & 0.009 \\
\hline Trochlear sulcus depth (mm) & 3.9 & 5.4 & 0.007 \\
\hline Insall-Salvati ratio & 1.2 & 1.1 & 0.8 \\
\hline Lateral patellofemoral angle (degree) & 9.1 & 9.7 & 0.6 \\
\hline Patellar translation (mm) & 4.1 & 3.9 & 0.2 \\
\hline Patellar alta:baja & $14: 11$ & $5: 4$ & 0.09 \\
\hline Patellar morphology ${ }^{a}$ (Wiberg's type A:B:C) & $2: 37: 11$ & $1: 27: 2$ & - \\
\hline
\end{tabular}

Abbreviations: CMP, chondromalacia patella; MRI, magnetic resonance imaging.

Note: Chi-square test used to obtain the $p$-value. Interobserver correlation coefficient was 0.92 .

Represent number of patients in the respective group.

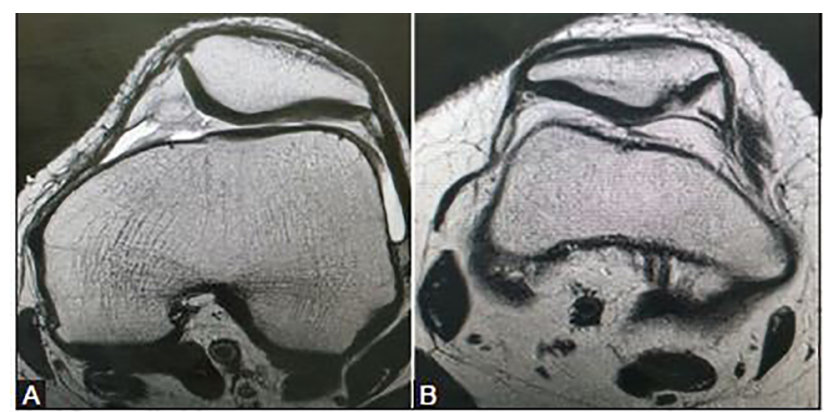

Fig. 8 (A) Axial T2-WI showing patellar articular cartilage changes in patients with trochlear dysplasia in early CMP. (B) Axial T2-WI showing patellar articular cartilage changes in patients with trochlear dysplasia in late CMP. CMP, chondromalacia patella; T2-WI, T2-weighted image.

There is plenty of research work done on trochlear morphology and PF pathology with contrasting results. ${ }^{23-27}$ The three-dimensional shape of the femoral trochlea is becoming the center of consideration in determining CMP. According to Ali et al, the trochlear morphology plays a key role in PF pathology and should always be taken into account while taking PF parameters. ${ }^{13}$ Our study favored multiple previous studies showing significant MRI results of lower trochlear depth and higher TSA are associated with higher cartilage defects ( - Table 3 and $\boldsymbol{- \text { Fig. }}$ 8). This finding supports our assumption which proposes that knees with flattened lateral trochlea are more likely to face CMP and we can identify patients at risk of developing CMP with higher TSA.

Patellar tilt can be assessed by lateral femoral angle which didn't show any association with the CMP in our study, supporting by the study of Aysin et al..$^{21}$ Contrary to our results, Yang et al and Tuna et al found correlation between lateral femoral angle and CMP with smaller lateral PF angle or medial opening of angle that is associated with higher probability of cartilage defect. ${ }^{10,28}$

The literature is muddled with multiple knee examination tests and we know that there is no single best clinical test to predict CMP. ${ }^{29}$ The low sensitivity and predictive value of Clark's sign as shown by Doberstein et al were also strengthened in our study than $50 \%$ of advanced CMP patient had positive Clark's sign. ${ }^{30}$ So, we are of this opinion that combining clinical test with clinical scores of KPSS or PFP scale (PFPS) can increase the predictability rate of CMP and may help to formulate a criteria to diagnose it more precisely. However, to verify this presumption, there is a need for extensive research with large series showing substantial data.

Articular cartilage defects (ACD) in the PF region are more common with various dysplastic shapes of the pat ella. ${ }^{14,31,32}$ However, a small number of investigations have revealed correlations between the shape of the patella and ACD in the PF region. Wiberg himself stated that type-A (I) and -B (II) shapes of patella are less destined to ACD in the PF region. ${ }^{44,32}$ Our study supported Gudas et al and Wiberg's classification system observing type II as most common variant and type $\mathrm{C}$ most closely associated with advanced CMP. Gudas et al observed type-II patella as most common variant and also concluded that the shape of the patella is an important anatomic parameter which may reflect the development of articular cartilage defects in the PF region. ${ }^{14}$ So, it can be anticipated that type-C (III) morphology can have a progressive course to CMP which further can be halted by proactive measures in the form chondroprotective drugs and strict adherence to "do's and don'ts" regimen for the knee care. 
Insall-Salvati ratio has been the matter of debate as a possible risk factor for CMP. ${ }^{33-37}$ Limited studies found an association between patella alta and CMP postulating that patella alta contributes to maltracking and PF mismatch. ${ }^{33,36}$ Other studies have shown no significant correlation between InsallSalvati ratio and CMP. ${ }^{21}$ Dowd and Bentley rather found association between patella alta and patellar instability but failed to find any correlation with CMP. ${ }^{34}$ In concordance with these findings, our study did not show any significant interconnection between the presence of CMP and Insall-Salvati ratio despite having higher ratio of patella alta to patella baja in advanced chondromalacia group.

\section{Limitations}

This study includes following limitations: static $\mathrm{Q}$ angle, nonfeasibility of standing position for MRI, medium cohort size, no follow-up, and no arthroscopy correlation.

\section{Future Considerations}

Further studies should have a longer follow-up with larger group specifically focusing on trochlear and patellar morphology and assessment of progression in CMP.

\section{Conclusion}

A holistic approach is required to interpret the multifactorial association of CMP. Our study concludes that a combination of $\mathrm{Q}$ angle, clinical examination, and PF scores can be used in the preliminary criteria to isolate CMP patients. These patients further can be directed for detailed radiological imaging of trochlear and patellar morphology to delineate underlying pathology, severity, and management. Furthermore, radiological criteria can be generated to predict progression of early to late CMP and to assess patients at risk of developing CMP. Lateral PF angle and Insall-Salvati ratio add little to the understanding of CMP pathology and may be skipped in routine PF assessment.

\section{Ethical Approval}

This study was approved by Ethics Committee and the ethical approval number is GGS/IEC//19/19.

\section{Financial Support and Sponsorship \\ None.}

\section{Conflicts of Interest}

There are no conflicts of interest.

\section{Acknowledgment}

Authors would like to thank Department of Social and Preventive Medicine.

\section{References}

1 Crossley KM, Stefanik JJ, Selfe J, et al. 2016 Patellofemoral pain consensus statement from the 4th International Patellofemoral Pain Research Retreat, Manchester. Part 1: terminology, definitions, clinical examination, natural history, patellofemoral osteoarthritis and patient-reported outcome measures. $\mathrm{Br}$ J Sports Med 2016;50(14):839-843
2 Roush JR, Curtis Bay R, Curtis Bay R. Prevalence of anterior knee pain in 18-35 year-old females. Int J Sports Phys Ther 2012;7(4):396-401

3 Ge Z, Li C, Heng BC, Cao G, Yang Z. Functional biomaterials for cartilage regeneration. J Biomed Mater Res A 2012;100(9):2526-2536

4 McCauley TR, Recht MP, Disler DG. Clinical imaging of articular cartilage in the knee. Semin Musculoskelet Radiol 2001;5(4):293-304

5 Crossley KM, Bennell KL, Cowan SM, Green S. Analysis of outcome measures for persons with patellofemoral pain: which are reliable and valid? Arch Phys Med Rehabil 2004;85(5):815-822

6 Bennell K, Bartam S, Crossley K, Green S. Outcome measures in patellofemoral pain syndrome: test retest reliability and inter-relationships. Phys Ther Sport 2000;1:32-41

7 Kujala UM, Jaakkola LH, Koskinen SK, Taimela S, Hurme M, Nelimarkka O. Scoring of patellofemoral disorders. Arthroscopy 1993;9(2):159-163

8 Chhabra A, Subhawong TK, Carrino JA. A systematised MRI approach to evaluating the patellofemoral joint. Skeletal Radiol 2011;40(4):375-387

9 Salzmann GM, Weber TS, Spang JT, Imhoff AB, Schöttle PB. Comparison of native axial radiographs with axial MR imaging for determination of the trochlear morphology in patients with trochlear dysplasia. Arch Orthop Trauma Surg 2010;130(3):335-340

10 Yang B, Tan H, Yang L, Dai G, Guo B. Correlating anatomy and congruence of the patellofemoral joint with cartilage lesions. Orthopedics 2009;32(1):20

11 Koskinen SK, Taimela S, Nelimarkka O, Komu M, Kujala UM. Magnetic resonance imaging of patellofemoral relationships. Skeletal Radiol 1993;22(6):403-410

12 Crema MD, Roemer FW, Marra MD, et al. Articular cartilage in the knee: current MR imaging techniques and applications in clinical practice and research. Radiographics 2011;31(1):37-61

13 Ali SA, Helmer R, Terk MR. Analysis of the patellofemoral region on MRI: association of abnormal trochlear morphology with severe cartilage defects. AJR Am J Roentgenol 2010;194(3):721-727

14 Gudas R, Šiupšinskas L, Gudaite A, et al. The patello-femoral joint degeneration and the shape of the patella in the population needing an arthroscopic procedure. Medicina (Kaunas 2018;54(2):21

15 Mattila VM, Weckström $M$, Leppänen $V$, Kiuru $M$, Pihlajamäki H. Sensitivity of MRI for articular cartilage lesions of the patellae. Scand J Surg 2012;101(1):56-61

16 Pihlajamäki HK, Kuikka PI, Leppänen VV, Kiuru MJ, Mattila VM. Reliability of clinical findings and magnetic resonance imaging for the diagnosis of chondromalacia patellae. J Bone Joint Surg Am 2010;92(4):927-934

17 Haim A, Yaniv M, Dekel S, Amir H. Patellofemoral pain syndrome: validity of clinical and radiological features. Clin Orthop Relat Res 2006;451(451):223-228

18 Emami MJ, Ghahramani MH, Abdinejad F, Namazi H. Q-angle: an invaluable parameter for evaluation of anterior knee pain. Arch Iran Med 2007;10(1):24-26

19 Patil S, White L, Jones A, Hui AC. Idiopathic anterior knee pain in the young. A prospective controlled trial. Acta Orthop Belg 2010;76(3):356-359

20 Türkmen F, Acar MA, Kacıra BK, et al. A new diagnostic parameter for patellofemoral pain. Int J Clin Exp Med 2015;8(7):11563-11566

21 Aysin IK, Askin A, Mete BD, Guvendi E, Aysin M, Kocyigit H. Askin. Investigation of relationship between anterior knee pain, chondromalacia patella and patellofemoral malalignment. Eurasian J Med 2018;50(1):28-33

22 Elson DW, Jones S, Caplan N. St Clair Gibson A, Stewart S, Kader DF. Clinically insignificant association between anterior knee 
pain and patellofemoral lesions which are found incidentally. Knee 2013;20(6):471-475

23 Duran S, Cavusoglu M, Kocadal O, Sakman B. Association between trochlear morphology and chondromalacia patella: an MRI study. Clin Imaging 2017;41:7-10

24 Mehl J, Feucht MJ, Bode G. Dovi-Akue D, Südkamp NP, Niemeyer P. Association between patellar cartilage defects and patellofemoral geometry: a matched-pair MRI comparison of patients with and without isolated patellar cartilage defects. Knee Surg Sports Traumatol Arthrosc 2016;24(3):838-846

25 McNally EG, Ostlere SJ, Pal C, Phillips A, Reid H, Dodd C. Assessment of patellar maltracking using combined static and dynamic MRI. Eur Radiol 2000;10(7):1051-1055

26 Özdemir M, Kavak RP. Chondromalacia patella among military recruits with anterior knee pain: Prevalence and association with patellofemoral malalignment. Indian J Orthop 2019;53(6):682-688

27 Thakkar RS, Del Grande F, Wadhwa V, et al. Patellar instability: CT and MRI measurements and their correlation with internal derangement findings. Knee Surg Sports Traumatol Arthrosc 2016;24(9):3021-3028

28 Tuna BK, Semiz-Oysu A, Pekar B, Bukte Y, Hayirlioglu A. The association of patellofemoral joint morphology with chondromalacia patella: a quantitative MRI analysis. Clin Imaging 2014;38(4):495-498

29 Loudon JK, Wiesner D, Goist-Foley HL, Asjes C, Loudon KL. Intrarater reliability of functional performance tests for subjects with patellofemoral pain syndrome. J Athl Train 2002;37(3):256-261

30 Doberstein ST, Romeyn RL, Reineke DM. The diagnostic value of the Clarke sign in assessing chondromalacia patella. J Athl Train 2008;43(2):190-196

31 de Lange-Brokaar BJE, Bijsterbosch J, Kornaat PR, et al. Radiographic progression of knee osteoarthritis is associated with MRIabnormalities in both the patellofemoral and tibiofemoral joint. Osteoarthritis Cartilage 2016;24(3):473-479

32 Kim HS, Yoo JH, Park NH, Chang JH, Ban YS, Song SH. Magnetic resonance imaging findings in small patella syndrome. Knee Surg Relat Res 2016;28(1):75-78

33 Insall J, Falvo KA, Wise DW. Chondromalacia patellae. a prospective study. J Bone Joint Surg Am 1976;58(1):1-8

34 Dowd GS, Bentley G. Radiographic assessment in patellar instability and chondromalacia patellae. J Bone Joint Surg Br 1986;68(2):297-300

35 Aglietti P, Cerulli G. Chondromalacia and recurrent subluxation of the patella: a study of malalignment, with some indications for radiography. Ital J Orthop Traumatol 1979;5(2):187-201

36 Lancourt JE, Cristini JA. Patella alta and patella infera. Their etiological role in patellar dislocation, chondromalacia, and apophysitis of the tibial tubercle. J Bone Joint Surg Am 1975;57(8):1112-1115

37 Marks KE, Bentley G. Patella alta and chondromalacia. J Bone Joint Surg Br 1978;60(1):71-73 